University of Nebraska - Lincoln

DigitalCommons@University of Nebraska - Lincoln

$7-2010$

Excited state geometry of photoactive yellow protein chromophore: A combined conductorlike polarizable continuum model and time-dependent density functional study

Yali Wang

University of Nebraska-Lincoln, yali.wang@huskers.unl.edu

Hui Li

University of Nebraska - Lincoln, hli4@unl.edu

Follow this and additional works at: https://digitalcommons.unl.edu/chemistryli

Part of the Chemistry Commons

Wang, Yali and Li, Hui, "Excited state geometry of photoactive yellow protein chromophore: A combined conductorlike polarizable continuum model and time-dependent density functional study" (2010). Hui Li Publications. 3.

https://digitalcommons.unl.edu/chemistryli/3

This Article is brought to you for free and open access by the Published Research - Department of Chemistry at DigitalCommons@University of Nebraska - Lincoln. It has been accepted for inclusion in Hui Li Publications by an authorized administrator of DigitalCommons@University of Nebraska - Lincoln. 


\title{
Excited state geometry of photoactive yellow protein chromophore: A combined conductorlike polarizable continuum model and time-dependent density functional study
}

\author{
Yali Wang and Hui $\mathrm{Li}^{\mathrm{a}}$ \\ Department of Chemistry, University of Nebraska-Lincoln, Lincoln, Nebraska 68588, USA
}

(Received 22 April 2010; accepted 18 June 2010; published online 19 July 2010)

\begin{abstract}
Analytic gradient of the combined conductorlike polarizable continuum model (CPCM) and time-dependent density functional theory method is derived and implemented. Due to the use of the fixed points with variable areas tessellation scheme, the excited state potential energy surfaces (PESs) are rigorously continuous and smooth. The CPCM/TD-B3LYP method is used to study an analog of the photoactive yellow protein chromophore, anionic thiomethyl $p$-coumaric acid $\left(\mathrm{TMpCA}^{-}\right)$. Although CPCM/TD-B3LYP method may not be accurate in predicting solvent effect on vertical excitation of $\mathrm{TMpCA}^{-}$, it may be used to predict redshiftings of emission maxima relative to absorption maxima with an accuracy of $\sim 0.1 \mathrm{eV}$. We also found that the excited trans-TMpCA${ }^{-}$tends to form a single bond twisted structure in the gas phase but a double bond twisted structure in aqueous solution. The TD-B3LYP minimum energy isomerization pathway shows a barrier of $3.6 \mathrm{kcal} / \mathrm{mol}$ in aqueous solution and $5.2 \mathrm{kcal} / \mathrm{mol}$ in the gas phase. The gas phase double bond twisted structure is trapped in a well of the excited state PES, with a depth of $\sim 20 \mathrm{kcal} / \mathrm{mol}(0.88 \mathrm{eV})$, in good agreement with an experimental value of $\sim 1 \mathrm{eV}$.

(c) 2010 American Institute of Physics. [doi:10.1063/1.3462248]
\end{abstract}

\section{INTRODUCTION}

Due to its low computational cost and relatively high accuracy for valence and singly excited states, the timedependent density functional theory (TDDFT) method developed by Casida et al. ${ }^{1}$ was extensively used in the past decade to study excited molecules. Recently Ziegler et $a .^{2}$ showed that TDDFT method can also be derived from a variational treatment. Van Caillie and $\mathrm{Amos}^{3}$ and Furche and Ahlrichs ${ }^{4}$ derived the analytic gradients of the TDDFT excitation energy using the Z-vector method originally developed by Handy and Schaefer, ${ }^{5}$ so molecular geometry optimization and molecular dynamics simulation on excited state potential energy surfaces (PESs) can be efficiently performed.

Solvent effect must be considered for molecules in condensed phases. Since full quantum mechanical $(\mathrm{QM})$ description of all solvent molecules is impractical, explicit molecular mechanical (MM) solvation models and implicit dielectric continuum solvation models are often used. The advantages and disadvantages of explicit and implicit solvation models are well known: Explicit models can provide specific intermolecular interactions but usually require expensive simulations; implicit models are much more efficient, but have no specific interactions. Polarizable solvation models are preferred in the study of excitation processes and dynamics on excited state PESs. This is due to the fact that the electronic excitation process is essentially instantaneous, and only the electron density of the solvent is able to quickly respond to the fast electron density change in the solute mol-

\footnotetext{
a) Author to whom correspondence should be addressed. Electronic mail: hli4@unl.edu.
}

ecule. A polarizable explicit model (i.e., a polarizable force field) can describe the fast electron density polarization by using, for example, induced dipoles, and the slow molecular polarization by reorienting the solvent molecules. A polarizable implicit model should also be able to describe electron polarization and molecular polarization separately. A few polarizable QM/MM methods for excited state study were developed or used by Öhrn and Karlström, ${ }^{6}$ Lin and Gao, ${ }^{7}$ Kongsted et al., ${ }^{8}$ Jensen et al.,${ }^{9}$ and Yoo et al. ${ }^{10}$ However, analytic gradient is not available for these methods so efficient geometry optimization and molecular dynamics simulation cannot be performed. Cossi and Barone ${ }^{11}$ developed a combined TDDFT and polarizable continuum model (PCM) method, for which Scalmani et al. ${ }^{12}$ derived and implemented analytic gradient. In the PCM/TDDFT method, solvent electronic polarization is described using optical (i.e., fast) dielectric constant while the total polarization (electronic+molecular) is described using static dielectric constant. The PCM [including D-PCM, ${ }^{13}$ integral equation formalism PCM, ${ }^{14,15}$ and conductorlike PCM or CPCM (Refs. 16 and 17)], the conductorlike screening models [COSMO (Ref. 18) and GCOSMO (Ref. 19)], the SS(V)PE (Ref. 20) models, and the SM $x$ models ${ }^{21}$ are popular implicit dielectric continuum solvation models. In PCM, COSMO, GCOSMO, and SS(V)PE, the electrostatic polarization interaction between the solute and the solvent is determined via the induced apparent surface charge method.

Geometry optimization and molecular dynamics simulations are meaningful only when there is a continuous and smooth PES, and only when the analytic gradient is accurate and can be evaluated efficiently. Recently we obtained rigorously continuous and smooth PESs and exact gradient for 
CPCM using the fixed points with variable areas [FIXPVA (Ref. 22)] tessellation scheme. Owning to its simplicity, the CPCM gradient can be evaluated exactly and efficiently (in terms of computer memory requirement and computing time) using FIXPVA. Very recently, Lange and Herbert ${ }^{23}$ and Scalmani and Frisch $^{24}$ also obtained continuous and smooth PESs for various versions of PCM.

In this work, we extend our FIXPVA tessellation scheme to the CPCM/TDDFT method, and derived and implemented the analytic gradient for it. Due to the use of FIXPVA, rigorously continuous and smooth PESs for excited states are guaranteed. Again, CPCM allows for an efficient evaluation of the exact CPCM/TDDFT gradient. To the best of our knowledge, this is the first time that rigorously continuous and smooth CPCM/TDDFT PESs are obtained.

Photoactive yellow protein (PYP) is a 125-residue photoreceptor found in the bacterium Ectothiprhodospira halophila. $^{25}$ The PYP chromophore is a deprotonated $p$-coumaric acid covalently bonded to Cys69 via a thioester linkage, and its phenolate oxygen is in hydrogen bonding with side chain hydroxyl groups of Tyr42 and Glu46. ${ }^{26}$ After blue light absorption [maximum $446 \mathrm{~nm}$ or $2.78 \mathrm{eV}$ (Ref. 25)], the chromophore in PYP undergoes an ultrafast transto-cis isomerization in the time scale of a few picoseconds. ${ }^{27}$ Due to its small size and thermal stability PYP has been a model system for extensive experimental studies. Some small derivatives of $p$-coumaric acid, as analogs of the PYP chromophore, have also been studied in the gas phase, aqueous solution, and PYP using experimental methods. ${ }^{28-36}$ Quantum chemical calculations have also been used to study the PYP chromophore. Of course, at the present time, only relatively small models (analogs) can be used in quantum chemical calculations. While most of these studied used complete active space self-consistent field (CASSCF) and complete active space second order perturbation theory (CASPT2) method, ${ }^{37-42}$ TDDFT was used to study vertical excitation of the PYP chromophore, for example, by Sergi et al. ${ }^{43}$ Groenhof et al. ${ }^{44}$ and Thompson et al. ${ }^{45}$ In addition, Levine et al. ${ }^{46}$ showed that the gas phase TDDFT method can be reliably applied to characterize a minimum energy isomerization pathway for neutral $p$-coumaric acid because the excited state along that pathway is dominated by single excitation. Therefore, TDDFT is a suitable theoretical tool for studying some important part of the PES of this chromophore. To the best of our knowledge, in literature there is only one PCM/TDDFT calculation ${ }^{47}$ for PYP performed by Chiba et al. using the fragment molecular orbital (FMO) scheme, and there is no PCM/TDDFT calculation for PYP chromophore analogs.

In this work we use the CPCM/TDDFT method to study an analog of the PYP chromophore, anionic thiomethyl $p$-coumaric acid $\left(\mathrm{TMpCA}^{-}\right.$, Fig. 1). This is probably the best analog of the PYP chromophore in terms of charge state and thioester linkage. It is also a model that is small enough for quantum chemical calculations and large enough to include most of the chromophore chemistry. Experimental data for $\mathrm{TMpCA}^{-}$in aqueous solution and PYP are available. ${ }^{28-31,35}$ CPCM/TDDFT is probably the most efficient quantum chemical method for the study of the equilibrium properties

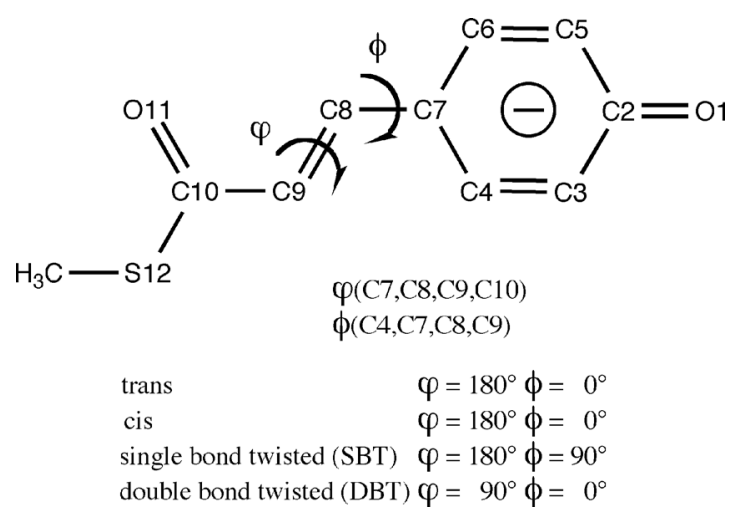

FIG. 1. Anionic thiomethyl $p$-coumaric acid $\left(\mathrm{TMpCA}^{-}\right)$.

of chromophores in solution. Although explicit dielectric continuum models are not generally good for describing protein matrix solvation due to the lacking of specific interactions, they can be used to obtain a quick and rough estimate of the protein solvation effect. We focus on comparing the CPCM/TDDFT results to spectroscopic data obtained in the gas phase and aqueous solution experiments.

The paper is organized as follows. In Sec. II, we briefly review the TDDFT and PCM/TDDFT theory, and then describe the formulas for calculating the CPCM/TDDFT gradient. In Sec. III, we describe the implementation of the CPCM/TDDFT code and the general computational method used in this study. In Sec. IV, we present and discuss the results of the PYP calculations, and compare them to experimental data. Finally in Sec. V, we conclude by highlighting the main results and findings.

\section{THEORY}

The central equation in the gas phase TDDFT method is

$$
\left(\begin{array}{ll}
\mathbf{A} & \mathbf{B} \\
\mathbf{B} & \mathbf{A}
\end{array}\right)\left(\begin{array}{l}
\mathbf{X} \\
\mathbf{Y}
\end{array}\right)=\omega\left(\begin{array}{cc}
1 & 0 \\
0 & -1
\end{array}\right)\left(\begin{array}{l}
\mathbf{X} \\
\mathbf{Y}
\end{array}\right)
$$

where $\mathbf{X}$ and $\mathbf{Y}$ are the linear response amplitudes in the excitation and de-excitation processes; $\omega$ is the excitation energy, $\mathbf{A}$ and $\mathbf{B}$ matrices are the orbital rotation Hessian,

$$
\begin{aligned}
& A_{i a s, j b s^{\prime}}=\delta_{i j} \delta_{a b} \delta_{s s^{\prime}}\left(\varepsilon_{a s}-\varepsilon_{i s}\right)+K_{i a s, j b s^{\prime}}, \\
& B_{i a s, j b s^{\prime}}=K_{i a s, b j s^{\prime}} .
\end{aligned}
$$

In Eq. (2) $i$ and $j$ represent occupied spin orbitals, $a$ and $b$ represent virtual spin orbitals, $\varepsilon_{a s}$ and $\varepsilon_{i s}$ represent spin orbital energies (later in the paper $\varepsilon$ is used to denote dielectric constants), $s$ and $s^{\prime}$ are spin indices, and $\mathbf{K}$ represents the coupling matrix, which contains the Coulomb term, DFT exchange-correlation term, and Hartree-Fock exchange terms in hybrid methods.

Cossi and Barone ${ }^{11}$ developed a PCM/TDDFT method in which PCM affects the TDDFT excitation energy in two ways. First, PCM implicitly affects the excited state by affecting the ground state DFT spin orbitals and their energies. Second, PCM explicitly affects the excitation process by affecting the $\mathbf{A}$ and $\mathbf{B}$ matrices, 


$$
\begin{aligned}
& A_{i a s, j b s^{\prime}}=\delta_{i j} \delta_{a b} \delta_{s s^{\prime}}\left(\varepsilon_{a s}-\varepsilon_{i s}\right)+K_{i a s, j b s^{\prime}}+G_{i a s, j b s^{\prime}}, \\
& B_{i a s, j b s^{\prime}}=K_{i a s, b j s^{\prime}}+G_{i a s, j b s^{\prime}},
\end{aligned}
$$

where the PCM term $G_{i a s, j b s^{\prime}}$ is the electrostatic interaction energy between the electrostatic potential created by the electron density $\psi_{i} \psi_{a}$ and the PCM charge induced by the electron density $\psi_{j} \psi_{b}$, or equivalently, the electrostatic interaction energy between the electrostatic potential created by the electron density $\psi_{j} \psi_{b}$ and the PCM charge induced by the electron density $\psi_{i} \psi_{a}$. $G_{i a s, j b s^{\prime}}$ is directly affected by the dielectric constant of the bulk solvent [see Eqs. (7) and (8)]. To study solvent-nonequilibrium vertical excitation processes in solution, the static dielectric constant of the solvent should be used in the ground state PCM/DFT calculation, but only the optical dielectric constant (i.e., the square of the refractive index) should be used in the PCM/TDDFT calculation. ${ }^{11,48}$ However, for PCM/TDDFT calculation of solvent-equilibrium properties of the exited state, the static dielectric constant should be used.

Equation (1) has solutions corresponding to various excited states. For a given state, the excitation energy $\omega$ is

$$
\omega=\frac{1}{2}\langle\mathbf{X}+\mathbf{Y}|\mathbf{A}+\mathbf{B}| \mathbf{X}+\mathbf{Y}\rangle+\frac{1}{2}\langle\mathbf{X}-\mathbf{Y}|\mathbf{A}-\mathbf{B}| \mathbf{X}-\mathbf{Y}\rangle
$$

The total energy of the excited state is the sum of the ground state DFT or PCM/DFT energy and the TDDFT or PCM/ TDDFT excitation energy $\omega$.

Furche and Ahlrichs ${ }^{4}$ extended Handy and Schaefer's Z-vector ${ }^{5}$ method to calculate the first derivative of the TDDFT excitation energy $\omega$ with respect to an atomic coordinate $x$,

$$
\begin{aligned}
\omega^{x}= & \frac{1}{2}\left\langle\mathbf{X}+\mathbf{Y}\left|(\mathbf{A}+\mathbf{B})^{x}\right| \mathbf{X}+\mathbf{Y}\right\rangle+\frac{1}{2}\left\langle\mathbf{X}-\mathbf{Y}\left|(\mathbf{A}-\mathbf{B})^{x}\right| \mathbf{X}-\mathbf{Y}\right\rangle \\
= & \sum_{\mu \nu s} P_{\mu \nu s}^{\Delta} h_{\mu \nu}^{x}-\sum_{\mu \nu s} W_{\mu \nu s^{\Delta}}^{\Delta} S_{\mu \nu}^{x}+\sum_{\mu \nu s \rho \sigma s^{\prime}} P_{\mu \nu s}^{\Delta} P_{\rho \sigma s^{\prime}}\left(f_{\mu \nu s, \rho \sigma s^{\prime}}^{\mathrm{XC}}\right)^{x} \\
& +\sum_{\mu \nu s \rho \sigma s^{\prime}} \Gamma_{\mu \nu s \rho \sigma s^{\prime}}^{\Delta}(\mu \nu \mid \rho \sigma)^{x} \\
& +\sum_{\mu \nu \rho \rho \sigma s^{\prime}}(X+Y)_{\mu \nu s}(X+Y)_{\rho \sigma s^{\prime}}\left(f_{\mu \nu s, \rho \sigma s^{\prime}}^{\mathrm{XC}}\right)^{x}
\end{aligned}
$$

where $\mu, \nu, \rho$, and $\sigma$ denote basis functions; $\mathbf{h}$ and $\mathbf{S}$ are the basis set one-electron and overlap integral matrices; $\mathbf{P}^{\Delta}$ is the relaxed one-particle difference density matrix; $\mathbf{P}$ is the ground state one-particle density matrix; $\mathbf{W}^{\Delta}$ is the energyweighted difference density matrix, which absorbs all terms involving the derivatives of the difference density matrix $\mathbf{P}^{\Delta}$; $\Gamma^{\Delta}$ is the two-particle difference density matrix; $f^{\mathrm{XC}}$ is the Hartree-Fock or DFT exchange-correlation kernel in the adiabatic approximation. The expression of $\Gamma^{\Delta}, \mathbf{T}^{\Delta}, \mathbf{Z}$, and $\mathbf{W}^{\Delta}$ can be found in literature. ${ }^{4}$
As shown by Scalmani et al., ${ }^{12}$ the gradients of the excitation energy in PCM/TDDFT can be obtained by adding the PCM terms $\boldsymbol{j}$ and $\mathbf{G}$ to the gas phase formula

$$
\begin{aligned}
\omega^{x}= & \sum_{\mu \nu s} P_{\mu \nu s}^{\Delta} h_{\mu \nu}^{x}-\sum_{\mu \nu s} W_{\mu \nu s^{\prime}}^{\Delta} S_{\mu \nu}^{x}+\sum_{\mu \nu s \rho \sigma s^{\prime}} P_{\mu \nu s}^{\Delta} P_{\rho \sigma s^{\prime}}\left(f_{\mu \nu s, \rho \sigma s^{\prime}}^{\mathrm{XC}}\right)^{x} \\
& +\sum_{\mu \nu s \rho \sigma s^{\prime}} \Gamma_{\mu \nu s \rho \sigma s^{\prime}}^{\Delta}(\mu \nu \mid \rho \sigma)^{x} \\
& +\sum_{\mu \nu s \rho \sigma s^{\prime}}(X+Y)_{\mu \nu s}(X+Y)_{\rho \sigma s^{\prime}}\left(f_{\mu \nu s, \rho \sigma s^{\prime}}^{\mathrm{XC}}\right)^{x} \\
& +\sum_{\mu \nu s} P_{\mu \nu s}^{\Delta}\left(j_{\mu \nu}\right)^{x}+\sum_{\mu \nu s \rho \sigma s^{\prime}} P_{\mu \nu s}^{\Delta} P_{\rho \sigma s^{\prime}}\left(G_{\mu \nu, \rho \sigma}\right)^{x} \\
& +\sum_{\mu \nu s \rho \sigma s^{\prime}}(X+Y)_{\mu \nu s}(X+Y)_{\rho \sigma s^{\prime}}\left(G_{\mu \nu, \rho \sigma}\right)^{x}
\end{aligned}
$$

where the PCM term $j_{\mu \nu}$ ( $\mu$ and $\nu$ are two basis functions) is the electrostatic interaction energy between the electrostatic potential created by the electron density $\mu \nu$ and the PCM charge induced by the nuclei, or equivalently, the electrostatic interaction energy between the electrostatic potential created by the nuclei and the PCM charge induced by the electron density $\mu \nu . G_{\mu \nu, \rho \sigma}$ has the same meaning as defined for Eq. (3), but in the basis set space.

The derivation of Eq. (6) is straightforward if one realizes that the PCM and DFT corrections to the Hartree-Fock energy and gradient are very similar. As a matter of fact, both PCM and DFT can be viewed as reaction field methods that modify electron-electron repulsion (both PCM and DFT) and electron-nucleus attraction (only PCM). Clearly, in Eq. (6), $j_{\mu \nu}$ corresponds to the one-electron nuclear charge integral in $h_{\mu \nu}$, and $G_{\mu \nu, \rho \sigma}$ corresponds to $f_{\mu \nu, \rho \sigma}^{\mathrm{XC}}$.

In PCM and similar continuum solvation models such as COSMO, GCOSMO, and SS(V)PE, induced apparent surface charge is used to describe the polarization interaction between a solute and the dielectric continuum solvent, and the continuous distribution of the induced surface charge is discretized into a finite number of point charges in self- and mutual interactions. Among various self- and mutual interaction descriptions, CPCM or equivalently, COSMO, is the simplest one, which allows for the most efficient numerical determination of the discretized point charges and subsequent gradient evaluation. In our previous work, we have obtained exact CPCM gradient for a variety of quantum chemical methods such as restricted closed-shell HartreeFock (RHF), restricted open-shell Hartree-Fock, unrestricted open-shell Hartree-Fock and their DFT analogs, and multiconfiguration self-consistent field method. ${ }^{22}$ Recently the author's group has also obtained exact CPCM gradient for the Møller-Plesset second order perturbation theory method (MP2) and the result will be published soon.

In CPCM and COSMO, a set of induced point charges, written as a vector $\mathbf{q}$, is uniquely determined by a set of electrostatic potentials at the solute surface tesserae, written as a vector $\mathbf{V}$, according to the following linear response equation:

$$
\mathbf{C} \cdot \mathbf{q}=-(1-1 / \varepsilon) \mathbf{V} .
$$


The elements of the matrix $\mathbf{C}$ are $\mathbf{C}_{i i}=1.07 \sqrt{4 \pi / a_{i}}$ and $\mathbf{C}_{i j}$ $=1 /\left|\mathbf{r}_{i}-\mathbf{r}_{j}\right|$, with $a_{i}$ being the area and $\boldsymbol{r}_{i}$ being the center coordinates of tessera $i . \varepsilon$ is the dielectric constant of the solvent. We note that scaling factors other than $(1-1 / \varepsilon)$ have also been suggested. ${ }^{16,18,49}$ The factor 1.07 for $\mathbf{C}_{i i}$ was first obtained by Klamt and Schuurmann. ${ }^{18}$

The CPCM charge q can be obtained by solving Eq. (7) via matrix inversion or iteratively. The total CPCM surface charge $\mathbf{q}$ can be determined separately for the solute electrons, $\mathbf{q}_{e}$, and nuclei, $\mathbf{q}_{N}$, using $\mathbf{V}_{e}$ and $\mathbf{V}_{N}$. In addition, CPCM induced surface charge can be determined for an electron density formed by the product of two molecular orbitals such as $\psi_{i} \psi_{a}$ (i.e., $\mathbf{q}_{i a}$ ), or two basis functions such as $\mu \nu$ (i.e., $\mathbf{q}_{\mu \nu}$ ). Therefore, $G_{i a s, j b s^{\prime}}$ in Eq. (3), $G_{\mu \nu, \rho \sigma}$ and $j_{\mu \nu}$ in Eq. (6) can be written as

$$
\begin{aligned}
& G_{i a s, j b s^{\prime}}=\left(\mathbf{V}_{i a s}\right)^{T} \mathbf{q}_{j b s^{\prime}}=\left(\mathbf{V}_{j b s^{\prime}}\right)^{T} \mathbf{q}_{i a s}, \\
& G_{\mu \nu, \rho \sigma}=\left(\mathbf{V}_{\mu \nu}\right)^{T} \mathbf{q}_{\rho \sigma}=\left(\mathbf{V}_{\rho \sigma}\right)^{T} \mathbf{q}_{\mu \nu}, \\
& j_{\mu \nu}=\left(\mathbf{V}_{\mu \nu}\right)^{T} \mathbf{q}_{N}=\left(\mathbf{V}_{N}\right)^{T} \mathbf{q}_{\mu \nu} .
\end{aligned}
$$

Here and hereafter the superscript $T$ denotes transpose.

In the following we derive the working equations for the implementation of Eq. (6) for CPCM/TDDFT. Because CPCM is a pure electrostatic method and is independent of the electronic spins, in the following equations and expressions we have omitted the spin indices for simplicity, and all density terms thus are the total density of alpha and beta electrons. The first five terms in Eq. (6) are identical to those in Eq. (5) and can be evaluated using the same procedure and computer code. The sixth term in Eq. (6) can be written as

$$
\begin{aligned}
\sum_{\mu \nu} P_{\mu \nu}^{\Delta}\left(j_{\mu \nu}\right)^{x}=\sum_{\mu \nu} P_{\mu \nu}^{\Delta}\left[\left(\mathbf{V}_{\mu \nu}\right)^{T} \mathbf{q}_{N}\right]^{x} & =\sum_{\mu \nu} P_{\mu \nu}^{\Delta}\left(\mathbf{V}_{\mu \nu}^{x}\right)^{T} \mathbf{q}_{N}+\sum_{\mu \nu} P_{\mu \nu}^{\Delta} \mathbf{V}_{\mu \nu}\left(-\frac{\varepsilon-1}{\varepsilon} \mathbf{C}^{-1} \mathbf{V}_{N}\right)^{x} \\
& =\sum_{\mu \nu} P_{\mu \nu}^{\Delta}\left(\mathbf{V}_{\mu \nu}^{x}\right)^{T} \mathbf{q}_{N}+\frac{\varepsilon-1}{\varepsilon} \sum_{\mu \nu}\left(P_{\mu \nu}^{\Delta} \mathbf{V}_{\mu \nu} \mathbf{C}^{-1} \mathbf{C}^{x} \mathbf{C}^{-1} \mathbf{V}_{N}-P_{\mu \nu}^{\Delta} \mathbf{V}_{\mu \nu} \mathbf{C}^{-1} \mathbf{V}_{N}^{x}\right) \\
& =\left(\mathbf{F}^{\Delta}\right)^{T} \mathbf{q}_{N}+\frac{\varepsilon}{\varepsilon-1}\left(\mathbf{q}^{\Delta}\right)^{T} \mathbf{C}^{x} \mathbf{q}_{N}+\left(\mathbf{q}^{\Delta}\right)^{T} \mathbf{V}_{N}^{x},
\end{aligned}
$$

where $\mathbf{F}^{\Delta}$ and $\mathbf{q}^{\Delta}$ are the electrostatic field and induced surface charge, respectively, due to the TDDFT excitation difference density,

$$
\begin{aligned}
& \mathbf{F}^{\Delta}=\sum_{\mu \nu} P_{\mu \nu}^{\Delta} \mathbf{V}_{\mu \nu}^{x}, \\
& \mathbf{q}^{\Delta}=-\frac{\varepsilon-1}{\varepsilon} \sum_{\mu \nu} P_{\mu \nu}^{\Delta} \mathbf{C}^{-1} \mathbf{V}_{\mu \nu} .
\end{aligned}
$$

Term $\left(\mathbf{F}^{\Delta}\right)^{T} \mathbf{q}_{N}$ in Eq. (11) represents the force imposed on $\mathbf{q}_{N}$ by the electrostatic field due to solute electronic difference density; the term $[\varepsilon /(\varepsilon-1)]\left(\mathbf{q}^{\Delta}\right)^{T} \mathbf{C}^{x} \mathbf{q}_{N}$ represents the electrostatic force between $\mathbf{q}_{N}$ and $\mathbf{q}^{\Delta}$; term $\left(\mathbf{q}^{\Delta}\right)^{T} \mathbf{V}_{N}^{x}$ represents the force imposed on $\mathbf{q}^{\Delta}$ by the electrostatic field due to solute electrons $\mathbf{V}_{N}^{x}$.

The seventh term in Eq. (6) can be written as

$$
\begin{aligned}
\sum_{\mu \nu \rho \sigma} P_{\mu \nu}^{\Delta} P_{\rho \sigma}\left(G_{\mu \nu, \rho \sigma}\right)^{x}=\sum_{\mu \nu \rho \sigma} P_{\mu \nu}^{\Delta} P_{\rho \sigma}\left[\left(\mathbf{V}_{\mu \nu}\right)^{T} \mathbf{q}_{\rho \sigma}\right]^{x}= & \sum_{\mu \nu \rho \sigma} P_{\mu \nu}^{\Delta} P_{\rho \sigma}\left(\mathbf{V}_{\mu \nu}^{x}\right)^{T} \mathbf{q}_{\rho \sigma}+\sum_{\mu \nu \rho \sigma} P_{\mu \nu}^{\Delta} P_{\rho \sigma} \mathbf{V}_{\mu \nu}\left(-\frac{\varepsilon-1}{\varepsilon} \mathbf{C}^{-1} \mathbf{V}_{\rho \sigma}\right)^{x} \\
= & \sum_{\mu \nu \rho \sigma} P_{\mu \nu}^{\Delta} P_{\rho \sigma}\left(\mathbf{V}_{\mu \nu}^{x}\right)^{T} \mathbf{q}_{\rho \sigma}+\frac{\varepsilon-1}{\varepsilon} \sum_{\mu \nu \rho \sigma}\left[P_{\mu \nu}^{\Delta} P_{\rho \sigma} \mathbf{V}_{\mu \nu} \mathbf{C}^{-1} \mathbf{C}^{x} \mathbf{C}^{-1} \mathbf{V}_{\rho \sigma}\right. \\
& \left.-P_{\mu \nu}^{\Delta} P_{\rho \sigma} \mathbf{V}_{\mu \nu} \mathbf{C}^{-1} \mathbf{V}_{\rho \sigma}^{x}\right]=\left(\mathbf{F}^{\Delta}\right)^{T} \mathbf{q}_{\mathrm{ele}}+\frac{\varepsilon}{\varepsilon-1}\left(\mathbf{q}^{\Delta}\right)^{T} \mathbf{C}^{x} \mathbf{q}_{\mathrm{ele}}+\left(\mathbf{q}^{\Delta}\right)^{T} \mathbf{V}_{\mathrm{ele}}^{x}
\end{aligned}
$$

The term $\left(\mathbf{F}^{\Delta}\right)^{T} \mathbf{q}_{\text {ele }}$ in Eq. (14) represents the force imposed on PCM induced charge $\mathbf{q}_{\text {ele }}$ by the electrostatic field due to solute electronic difference density; the term $[\varepsilon /(\varepsilon-1)]$ $\times\left(\mathbf{q}^{\Delta}\right)^{T} \mathbf{C}^{x} \mathbf{q}_{\text {ele }}$ represents the electrostatic force between $\mathbf{q}_{\text {ele }}$ and $\mathbf{q}^{\Delta}$; the term $\left(\mathbf{q}^{\Delta}\right)^{T} \mathbf{V}_{\text {ele }}^{x}$ represents the force imposed on $\mathbf{q}^{\Delta}$ by the electrostatic field due to solute electrons $\mathbf{V}_{\text {ele }}^{x}$.

The eighth term in Eq. (6) can be written as

$$
\begin{aligned}
& \sum_{\mu \nu \rho \sigma}(X+Y)_{\mu \nu}(X+Y)_{\rho \sigma}\left(G_{\mu \nu, \rho \sigma}\right)^{x} \\
& \quad=2\left(\mathbf{F}_{X Y}\right)^{T} \mathbf{q}_{X Y}+\frac{\varepsilon}{\varepsilon-1}\left(\mathbf{q}_{X Y}\right)^{T} \mathbf{C}^{x} \mathbf{q}_{X Y}
\end{aligned}
$$

where $\mathbf{F}_{X Y}$ and $\mathbf{q}_{X Y}$ are the electrostatic field and induced 
surface charge, respectively, due to the TDDFT transition state density $(X+Y)_{\mu \nu}$,

$$
\begin{aligned}
& \mathbf{F}_{X Y}=\sum_{\mu \nu}(X+Y)_{\mu \nu} \mathbf{V}_{\mu \nu}^{x}, \\
& \mathbf{q}_{X Y}=-\frac{\varepsilon-1}{\varepsilon} \sum_{\mu \nu}(X+Y)_{\mu \nu} \mathbf{C}^{-1} \mathbf{V}_{\mu \nu} .
\end{aligned}
$$

The term $2\left(\mathbf{F}_{X Y}\right)^{T} \mathbf{q}_{X Y}$ in Eq. (15) represents the force imposed on the transition state density induced charge $\mathbf{q}_{X Y}$ by the electrostatic field due to the transition state density $(X+Y)_{\mu \nu}$; the term $[\varepsilon /(\varepsilon-1)]\left(\mathbf{q}_{X Y}\right)^{T} \mathbf{C}^{x} \mathbf{q}_{X Y}$ represents the force between $\mathbf{q}_{X Y}$.

The evaluation of Eqs. (11), (14), and (15) requires $\mathbf{C}^{x}$, which involves the derivatives of the areas and coordinates of the tesserae with respect to the atomic coordinate $x$. Recently, $\mathrm{Su}$ and $\mathrm{Li}^{22}$ implemented a tessellation scheme called FIXPVA for CPCM. In FIXPVA the tessera areas are smooth functions of their distances to neighboring spheres so rigorously continuous and smooth potential energy surfaces, as well as exact analytic gradients, can be obtained.

\section{COMPUTATIONAL METHODOLOGY}

The CPCM/TDDFT gradient has been implemented in the GAMESS (Ref. 50) package (dated 25 March 2010 and released on 11 May 2010) by the authors based on the TDDFT (both energy and gradient) and CPCM/TDDFT (energy only) code previously implemented by Chiba et al. ${ }^{51}$ and the FIXPVA tessellation code implemented by Su and $\mathrm{Li}^{22}$ The CPCM code was previously implemented by $\mathrm{Li}$ and Jensen ${ }^{17}$ on the basis of the PCM program originally implemented by Mennucci and co-workers. ${ }^{14,52,53}$ Tests with various molecules and basis sets show that using the FIXPVA tessellation scheme and default settings in GAMESS, the accuracy of the CPCM/TDDFT analytic gradients is typically 5 $\times 10^{-6}$ a.u. as compared to numerical gradients obtained via finite double-displacement with step size of $\pm 0.001 \mathrm{bohr}$. Similar errors can be seen in the gas phase DFT and TDDFT calculations because they arise from the finite grid points in these methods. Geometry optimization, numerical Hessian calculation, and molecular dynamics simulation can be performed. In GAMESS, in order to improve geometry optimization convergence, the DFT and PCM gradients are subject to a projection that eliminates rotational gradients (torques) artificially exerted on the molecules by the DFT grid points and the PCM surface tesserae. The projection can typically alter the gradients by $\sim 10^{-4}$ a.u. To obtain the original gradients one can turn off the projection by changing the GAMESS source code in $\operatorname{grd} 2 a . s r c$. In addition, we note that the CPCM/TDDFT code also works for the time-dependent Hartree-Fock (a.k.a. random phase approximation) method when the density functionals are replaced by the HartreeFock exchange energy.

The wave function of the singlet ground state and a few singlet excited states of $\mathrm{TMpCA}^{-}$(Fig. 1) were calculated with the B3LYP (Ref. 54) and TD-B3LYP methods, respectively. The aug-cc-pVDZ (denoted as aDZ) ${ }^{55}$ were used in all the calculations unless specifically indicated. In CPCM cal- culation, static dielectric constants of 78.39 and 2.0 were used to represent bulk water and the PYP environment around the chromophore. Using 2.0 for PYP is reasonable because it is a highly rigid protein, as suggested by Thompson et al. ${ }^{45}$ The optical dielectric constant 1.776 was used for bulk water. The same value 1.776 was used for protein matrix. This is reasonable because, for example, benzene and ethanol have optical dielectric constants $\sim 2.0$. A few tests show that the excitation energies are very insensitive to the optical dielectric constant in the range of 1.8-2.2. We use the same value in favor of direct comparisons. Spheres with radii of $2.124,1.908$, and $2.520 \AA$ were used for $\mathrm{C}, \mathrm{O}$, and $\mathrm{S}$ atoms, respectively, to define the molecular cavity (i.e., the simplified united atomic radii for Hartree-Fock option in which $\mathrm{H}$ atoms have no contribution to the surface), and the surface tessellation scheme FIXPVA was used with 240 initial tesserae per sphere. It is noted that although the absolute solvation free energy calculated with CPCM is sensitive to the radii, the excitation energy is not. The induced surface charges were determined by a semi-iterative procedure ${ }^{53,56}$ with no charge renormalization. Only the electrostatic interaction was considered; cavitation, dispersion, and repulsion terms were not considered. For comparison, corresponding gas phase B3LYP and TD-B3LYP calculations (no CPCM) were also performed. In both the singlet ground state B3LYP and the singlet excited state TD-B3LYP calculations 96 radial and 302 Lebedev angular grid points were used. Geometry optimization was performed with the $\mathrm{B} 3 \mathrm{LYP} / \mathrm{aDZ}$ and TD-B3LYP/aDZ methods in the gas phase and solution (with CPCM). Although a few geometries (either minima or saddle points) should have $\mathrm{C}_{\mathrm{s}}$ symmetry, no symmetry was actually imposed in all calculations. This is partially due to the FIXPVA tessellation scheme, which does not have symmetry for randomly orientated molecules.

Here we report the timings for the gas phase TD-B3LYP/ $\mathrm{aDZ}$ and CPCM $(\varepsilon=78.39) / \mathrm{TD}-\mathrm{B} 3 \mathrm{LYP} / \mathrm{aDZ}$ single point $\mathrm{S}_{1}$ energy and gradient calculations of the trans isomer of $\mathrm{TMpCA}^{-}$: The overall wall-clock timings using an eight-core node are 3428.0 and 3248.9 s, respectively. Therefore, adding CPCM effect actually decreases the timing. This is due to the difference in the ground state B3LYP self-consistent field calculation: The gas phase case took 32 iterations while the CPCM case only took 23 steps because solvent effect can somehow improve the convergence. If only the TDDFT step (excitation energy iteration and Z-vector iteration) is considered, the wall-clock timings are 2334.1 and $2355.2 \mathrm{~s}$. Clearly, including CPCM solvent effect into the TDDFT calculation only causes a marginal increase in the computation time.

\section{RESULTS AND DISCUSSION}

\section{A. Ground state}

The geometries of the ground state trans and cis isomers were optimized in the gas phase with the B3LYP method, and in solvent with the CPCM $(\varepsilon=2.0$ and 78.39)/B3LYP method. After optimization, both the trans and cis isomers show planar geometries (Fig. 1). They should have $\mathrm{C}_{\mathrm{s}}$ symmetry but in all the calculations no symmetry was imposed. As shown in Table I, the ground state trans and cis isomers 
TABLE I. Bond lengths $(\AA)$ in $\mathrm{TMpCA}^{-}$at five geometries optimized on the ground state and excited state potential energy surfaces (Fig. 1).

\begin{tabular}{|c|c|c|c|c|c|c|c|c|c|}
\hline Method & $\mathrm{O} 1=\mathrm{C} 2$ & $\mathrm{C} 2-\mathrm{C} 3$ & $\mathrm{C} 3=\mathrm{C} 4$ & $\mathrm{C} 4-\mathrm{C} 7$ & $\mathrm{C} 7-\mathrm{C} 8$ & $\mathrm{C} 8=\mathrm{C} 9$ & $\mathrm{C} 9-\mathrm{C} 10$ & $\mathrm{C} 10=\mathrm{O} 11$ & $\mathrm{C} 10-\mathrm{S} 12$ \\
\hline \multicolumn{10}{|c|}{ Ground trans } \\
\hline$\varepsilon=1.00$ & 1.258 & 1.457 & 1.375 & 1.431 & 1.420 & 1.384 & 1.434 & 1.229 & 1.861 \\
\hline$\varepsilon=2.00$ & 1.265 & 1.453 & 1.378 & 1.429 & 1.425 & 1.380 & 1.440 & 1.232 & 1.835 \\
\hline$\varepsilon=78.39$ & 1.278 & 1.446 & 1.382 & 1.425 & 1.432 & 1.375 & 1.448 & 1.235 & 1.816 \\
\hline \multicolumn{10}{|c|}{ Excited trans } \\
\hline$\varepsilon=1.00$ & 1.271 & 1.456 & 1.392 & 1.413 & 1.482 & 1.376 & 1.453 & 1.237 & 1.844 \\
\hline$\varepsilon=2.00$ & 1.275 & 1.454 & 1.387 & 1.420 & 1.471 & 1.380 & 1.453 & 1.244 & 1.824 \\
\hline$\varepsilon=78.39$ & 1.275 & 1.453 & 1.381 & 1.430 & 1.445 & 1.401 & 1.439 & 1.255 & 1.820 \\
\hline \multicolumn{10}{|c|}{ Excited SBT } \\
\hline$\varepsilon=1.00$ & 1.267 & 1.449 & 1.382 & 1.418 & 1.499 & 1.382 & 1.430 & 1.254 & 1.860 \\
\hline$\varepsilon=2.00$ & 1.265 & 1.452 & 1.380 & 1.422 & 1.490 & 1.389 & 1.423 & 1.259 & 1.858 \\
\hline$\varepsilon=78.39$ & 1.262 & 1.455 & 1.376 & 1.428 & 1.480 & 1.396 & 1.418 & 1.266 & 1.847 \\
\hline \multicolumn{10}{|c|}{ Excited DBT } \\
\hline$\varepsilon=1.00$ & 1.270 & 1.454 & 1.375 & 1.445 & 1.397 & 1.482 & 1.428 & 1.228 & 1.855 \\
\hline$\varepsilon=2.00$ & 1.276 & 1.451 & 1.377 & 1.442 & 1.402 & 1.473 & 1.432 & 1.231 & 1.834 \\
\hline$\varepsilon=78.39$ & 1.290 & 1.446 & 1.380 & 1.439 & 1.407 & 1.464 & 1.437 & 1.236 & 1.823 \\
\hline \multicolumn{10}{|c|}{ Ground cis } \\
\hline$\varepsilon=1.00$ & 1.257 & 1.458 & 1.372 & 1.438 & 1.419 & 1.397 & 1.431 & 1.227 & 1.875 \\
\hline$\varepsilon=2.00$ & 1.265 & 1.454 & 1.374 & 1.435 & 1.424 & 1.393 & 1.436 & 1.229 & 1.858 \\
\hline$\varepsilon=78.39$ & 1.277 & 1.447 & 1.379 & 1.431 & 1.433 & 1.386 & 1.446 & 1.233 & 1.825 \\
\hline
\end{tabular}

have very similar bond lengths, so only the bond lengths of the trans isomer are discussed here. The phenolate $\mathrm{O} 1=\mathrm{C} 2$ distance is $1.258 \AA$, a typical double bond length. The phenolate ring shows a clear quinonic alternation in bond lengths. In general, CPCM aqueous $(\varepsilon=78.39)$ solvation makes the double bonds slightly longer and the single bonds slightly shorter, all by $\sim 0.01 \AA$. There are two relatively large changes: the $\mathrm{O} 1=\mathrm{C} 2$ double bond is elongated by $0.02 \AA$, and the $\mathrm{C} 10-\mathrm{S} 12$ single bond is shortened by 0.05
$\AA$. In both gas phase and solution, the electronic energy (excluding zero point energy, vibration and rotation energy) of the trans isomer is lower than the cis isomer by $\sim 6 \mathrm{kcal} / \mathrm{mol}$ (Table II).

\section{B. Vertical excitation energy}

Based on the equilibrium geometries of the ground state trans and cis isomers, the vertical excitation energies of the

TABLE II. Ground state and excited state energies, excitation energies $\omega$, and oscillator strengths $f$ calculated for TMpCA ${ }^{-}$at five geometries optimized on the ground state and excited state potential energy surfaces.

\begin{tabular}{|c|c|c|c|c|c|}
\hline Geometry & Ground trans & Excited trans & Excited SBT & Excited DBT & Ground cis \\
\hline \multicolumn{6}{|c|}{$\varepsilon=1.00$} \\
\hline Ground (a.u.) & -934.90626 & -934.90184 & -934.86310 & -934.84340 & -934.89650 \\
\hline Excited (a.u.) & -934.78959 & -934.79293 & -934.84555 & -934.82921 & -934.78017 \\
\hline$\omega(\mathrm{eV})$ & 3.17 & 2.96 & 0.48 & 0.39 & 3.17 \\
\hline$f$ & 1.04 & 0.88 & 0.00 & 0.00 & 0.88 \\
\hline \multicolumn{6}{|c|}{$\varepsilon=2.00$} \\
\hline Ground (a.u.) & -934.94387 & -934.94034 & -934.90397 & -934.88161 & -934.93409 \\
\hline Excited (a.u.) & $-934.83391^{\mathrm{a}}$ & $-934.83761^{\mathrm{b}}$ & $-934.87117^{\mathrm{b}}$ & $-934.86312^{b}$ & $-934.82371^{\mathrm{a}}$ \\
\hline$\omega(\mathrm{eV})$ & $2.99^{\mathrm{a}}$ & $2.80^{\mathrm{b}}$ & $0.89^{\mathrm{b}}$ & $0.50^{\mathrm{b}}$ & $3.00^{\mathrm{a}}$ \\
\hline$f$ & 1.17 & 1.06 & 0.00 & 0.00 & 1.00 \\
\hline \multicolumn{6}{|c|}{$\varepsilon=78.39$} \\
\hline Ground (a.u.) & -934.98449 & -934.98249 & -934.94659 & -934.92094 & -934.97504 \\
\hline Excited (a.u.) & $-934.87327^{\mathrm{a}}$ & $-934.88540^{b}$ & $-934.89556^{\mathrm{b}}$ & $-934.89841^{\mathrm{b}}$ & $-934.86355^{\mathrm{a}}$ \\
\hline$\omega(\mathrm{eV})$ & $3.03^{\mathrm{a}}$ & $2.64^{\mathrm{b}}$ & $1.39^{\mathrm{b}}$ & $0.61^{\mathrm{b}}$ & $3.03^{\mathrm{a}}$ \\
\hline$f$ & 1.14 & 1.32 & 0.00 & 0.00 & 0.95 \\
\hline
\end{tabular}

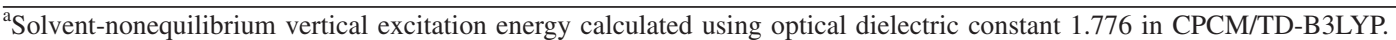

${ }^{\mathrm{b}}$ Solvent-equilibrium excitation energy calculated using static dielectric constants (2.0 or 78.39) in CPCM/TD-B3LYP. 
low-lying singlet states were calculated with the TD-B3LYP method, in the gas phase and solutions. In all of the cases occurred in the current study, the largest single excitation amplitude, typically above 0.99 , is that from the highest occupied molecular orbital (HOMO) to the lowest unoccupied molecular orbital (LUMO).

In the gas phase, according to the calculation, the trans isomer shows a $3.17 \mathrm{eV} \mathrm{HOMO} \rightarrow$ LUMO transition $\left(\mathrm{S}_{0} \rightarrow \mathrm{S}_{2}\right)$ with an oscillator strength of 1.04. All other transitions have almost zero oscillator strengths. For example, the $3.08 \mathrm{eV} \mathrm{HOMO} \rightarrow \mathrm{LUMO}+1$ transition $\left(\mathrm{S}_{0} \rightarrow \mathrm{S}_{1}\right)$ has an oscillator strength of 0.0002 . Similarly, for the cis isomer only the $3.17 \mathrm{eV} \mathrm{HOMO} \rightarrow$ LUMO transition $\left(\mathrm{S}_{0} \rightarrow \mathrm{S}_{3}\right)$ is significant (oscillator strength of 0.88 ), the $S_{0} \rightarrow S_{1}$ (3.10 $\mathrm{eV})$, and the $\mathrm{S}_{0} \rightarrow \mathrm{S}_{2}(3.15 \mathrm{eV})$ transitions, which correspond to $\mathrm{HOMO} \rightarrow \mathrm{LUMO}+1$ and $\mathrm{HOMO}-1 \rightarrow$ LUMO excitations, respectively, both have almost zero oscillator strengths. Orbital visualization shows that the HOMO and LUMO from the ground state B3LYP calculation are delocalized $\pi$ and $\pi^{*}$ orbitals, respectively, in accordance with TDDFT calculations in literature. ${ }^{45,57}$ The experimental absorption maxima of the gas phase trans- and cis-TMpCA ${ }^{-}$are not available. Nielsen et al. ${ }^{32}$ observed that anionic trans-thiophenyl p-coumarate (i.e., trans- $\mathrm{pCT}^{-}$), which is similar to trans-TMpCA ${ }^{-}$, shows an absorption maximum of $2.70 \mathrm{eV}$ in the gas phase. Because trans-TMpCA ${ }^{-}$is very similar to trans- $\mathrm{pCT}^{-}$, the gas phase absorption maximum of trans-TMpCA ${ }^{-}$can be estimated as $2.70 \mathrm{eV}$. If this is true, the current B3LYP value is $0.5 \mathrm{eV}$ too high. We also performed TD-B3LYP/aug-cc-pVTZ (Ref. 55) single point energy calculations and obtained a value differing by only $0.003 \mathrm{eV}$. Earlier, Thompson et al..$^{45}$ obtained $\sim 3.1 \mathrm{eV}$ using a TDDFT method. González et al. ${ }^{57}$ obtained $3.32 \mathrm{eV}$ with the TD-B3LYP/cc-pVTZ method (note that the B3LYP in GAMESS is different from what they used), and made the point that it is the intrinsic error in the TD-B3LYP methods that caused the errors in the calculated excitation energies. Based on the chromophore geometry in a crystal structure, Molina and Merchan ${ }^{38}$ performed multistate-CASPT2 calculation for trans-TMpCA ${ }^{-}$and obtained $2.58 \mathrm{eV}$. Gromov et $a l .{ }^{40}$ performed CC2/SV(P) and EOM-CCSD/6-31G(d) calculations for trans-TMpCA ${ }^{-}$cut from an $\mathrm{x}$-ray structure $[\mathrm{H}$ atoms optimized with RHF/6-31G(d)] and obtained 2.89 and $3.18 \mathrm{eV}$, respectively. Recently, Ma et al. ${ }^{58}$ performed manybody Green's function theory calculation and obtained 2.80 eV.

In solutions, both the trans and cis isomers show $\mathrm{HOMO} \rightarrow$ LUMO transitions similar to those in the gas phase. Due to the CPCM solvation, these transitions are all $\mathrm{S}_{0} \rightarrow \mathrm{S}_{1}$, and are all redshifted by $0.1-0.2 \mathrm{eV}$ as compared to those in the gas phase (Table II). According to the calculation, the trans and cis isomers show almost identical vertical excitation energies that differ by only $\sim 0.01 \mathrm{eV}$ in both the gas phase and solutions. González et al. ${ }^{57}$ obtained $3.32 \mathrm{eV}$ from a gas phase B3LYP/cc-pVTZ calculation for trans-TMpCA ${ }^{-}$, and $3.21 \mathrm{eV}$ from a QM/MM style B3LYP/ cc-pVTZ calculation for trans-TMpCA- in PYP. So, in their QM/MM calculation, the protein interaction redshifts the absorption maximum by $0.11 \mathrm{eV}$, in qualitative agreement with the current CPCM result. Chiba et al. obtained similar redshifting ongoing from the gas phase to protein using the FMO based TDDFT and CPCM/TDDFT methods. ${ }^{47,59}$ It is not clear what is the exact shifting ongoing from the gas phase to PYP or a low dielectric organic solvent. The best estimation based on the experimental value $2.70 \mathrm{eV}$ for trans- $\mathrm{pCT}^{-}$and $2.78 \mathrm{eV}$ for the chromophore in PYP is that there is a small blueshifting of $0.08 \mathrm{eV}^{32}$

In aqueous solution, the trans and cis isomers show $\mathrm{HOMO} \rightarrow \mathrm{LUMO} \quad\left(\mathrm{S}_{0} \rightarrow \mathrm{S}_{1}\right)$ vertical excitation energies of 3.03 and $3.03 \mathrm{eV}$ (with oscillator strengths of 1.14 and 0.95 ), respectively. Therefore, the CPCM/TD-B3LYP method predicts a redshifting of $\sim 0.1 \mathrm{eV}$ for both the trans and cis isomers. Nielsen et al. ${ }^{32}$ reported a $0.44 \mathrm{eV}$ blueshifting for trans-pCT ${ }^{-}$(gas phase of $2.70 \mathrm{eV}$ and aqueous of $3.14 \mathrm{eV}$ ). Because trans- $\mathrm{TMpCA}^{-}$is very similar to trans-pCT${ }^{-}$, there should be a similar blueshifting for trans-TMpCA${ }^{-}$. Indeed, Larsen et $a l .{ }^{28}$ reported that in aqueous solution $(\mathrm{pH}=10.5)$ the trans isomer has an absorption maximum of $3.22 \mathrm{eV}$, which is blueshifted by $0.44 \mathrm{eV}$ from the protein. As discussed before, the gas phase value and protein value should be very similar.

Clearly, CPCM/TD-B3LYP cannot predict the correct blueshifting of the absorption maximum on going from the gas phase to aqueous solution. This is not surprising because CPCM and similar continuum solvation models do not contain specific and dynamic solvent interactions such as hydrogen bonding. Vertical excitation is a fast process that should be calculated with instantaneous solute and solvent configurations in an ensemble. Despite this deficiency, in the following subsection we show that CPCM/TDDFT can be used to predict equilibrium molecular geometries on the excited state PESs and estimate the redshifting of the emission energy.

\section{Relaxation and vertical emission}

After vertical excitation, which takes only a few femtoseconds, the molecular geometry will relax on the excited state potential energy surface. Vertical emission may occur at various stages in the relaxation process. The initial intramolecular vibrational relaxation has a timescale around 10-20 $\mathrm{fs}$, and usually completes in $\sim 50 \mathrm{fs}$, resulting in energy redistribution in the molecule. Subsequent intramolecular rotational relaxation, if any, occurs in a timescale of $\sim 1$ ps.

The gas phase excited state equilibrium geometry of the trans isomer was optimized with the TD-B3LYP method based on the B3LYP optimized gas phase ground state geometry, which is planar (virtually of $\mathrm{C}_{\mathrm{s}}$ symmetry). It turned out that the planar geometry of the gas phase excited trans isomer is a saddle point and the optimization automatically leads to the $\mathrm{C} 7-\mathrm{C} 8$ single bond twisted geometry. However, before any twisting occurs, the geometry was already optimized to a point with a maximum gradient of 1.178 $\times 10^{-4}$ a.u. and a root mean square gradient of 0.441 $\times 10^{-4}$ a.u. This geometry is taken as the optimized trans (planar) geometry in the gas phase. After optimization in the gas phase, the single bonds in the phenolate ring tend to be shorter, and the double bond lengths tend to be longer. The $\mathrm{C} 7-\mathrm{C} 8$ single bond elongates by $0.062 \AA$; the $\mathrm{C} 8=\mathrm{C} 9$ 
alkyl double bond shortens by $0.008 \AA$; the $\mathrm{C} 9-\mathrm{C} 10$ single bond elongates by $0.019 \AA$; the $\mathrm{C} 10=011$ carbonyl double bond elongates by $0.008 \AA$; the $\mathrm{C} 10-\mathrm{S} 12$ single bond shortens by $0.017 \AA$. The calculated vertical emission energy from the excited state equilibrium geometry of the trans isomer in the gas phase is redshifted by $0.21 \mathrm{eV}$ as compared to the calculated vertical excitation energy (Table II). After the initial vibrational relaxation, intramolecular rotation or torsion relaxation may occur, as to be discussed in the next subsection.

In solution, solvent molecules will respond to the excitation caused by electron density change in different timescales. Solvent electronic polarization is essentially instantaneous (a few femtoseconds), and has been considered in the vertical excitation process. Solvent molecular vibration, rotation and translation motions, or molecular polarization occur in various timescales. For example, the measurements of Larsen et al. of $\mathrm{TMpCA}^{-}$in aqueous solution revealed that bulk water shows 100 and 500 fs molecular polarization timescales. ${ }^{29}$ Here we consider the case where the bulk water has established a new equilibrium with the relaxed excited geometries, corresponding to $\sim 2$ ps after vertical excitation. The excited state equilibrium geometry of the trans isomer in aqueous solution was optimized with the CPCM/TD-B3LYP method based on the CPCM/B3LYP optimized ground state geometry, which are planar and virtually of $\mathrm{C}_{\mathrm{s}}$ symmetry. CPCM solvation stabilizes the planar geometry of the excited state so it becomes a minimum point on the potential energy surface. Compared to those in the ground state geometries, the bond lengths in the excited state in aqueous solution are changed by $0.003-0.037 \AA$. For example, the $\mathrm{C} 7-\mathrm{C} 8$ single bond is shortened by $0.037 \AA$ and the $\mathrm{C} 8=\mathrm{C} 9$ double bond is lengthened by $0.025 \AA$ (Table I). In aqueous solution, the calculated solvent-equilibrium excitation energy of the trans isomer is $2.64 \mathrm{eV}$ (Table II). This solvent-equilibrium excitation energy corresponds to a transition from solvent-equilibrium ground state to solventequilibrium excited state, or the emission energy of the reverse process. However, it cannot be directly compared to the vertical emission energy from solvent-equilibrium excited state to solvent-nonequilibrium ground state. The current CPCM/TDDFT implementation cannot calculate the nonequilibrium vertical emission energy. Alternatively, we performed a separate CPCM/TD-B3LYP solventnonequilibrium excitation energy calculation for the trans isomer and obtained $2.92 \mathrm{eV}$. Therefore, the solvent molecular polarization energy is $0.28 \mathrm{eV}$ for the ground-to-excited density change. If we assume that the solvent molecular polarization energy for the excited-to-ground density change is the same as that for the ground-to-excited density change, i.e., $0.28 \mathrm{eV}$, the vertical emission energy can be estimated as $2.36 \mathrm{eV}$. This value is redshifted by $0.67 \mathrm{eV}$ from the calculated vertical excitation energy $3.03 \mathrm{eV}$ (Table II), and is comparable to the experimental value of $0.74 \mathrm{eV}$ reported by Larsen et $_{\text {al. }}{ }^{28}$ (vertical excitation $3.22 \mathrm{eV}$ or $385 \mathrm{~nm}$ versus $2.48 \mathrm{eV}$ or $500 \mathrm{~nm}$ at $\sim 2 \mathrm{ps}$ ). Such an agreement suggests that the equilibrium geometry and the solvent molecular polarization energy estimated by the CPCM/TD-B3LYP method are reasonably good.
Although in general CPCM is not valid for protein matrix solvation, it is worth comparing the CPCM/TDDFT results to experimental data. The CPCM $(\varepsilon=2.0) /$ TD-B3LYP calculated solvent-equilibrium excitation energy of the trans isomer is $2.80 \mathrm{eV}$ (Table II). A separate solventnonequilibrium excitation energy calculation shows that the solvent molecular polarization energy is $0.027 \mathrm{eV}$ for the ground-to-excited density change. Using the same assumption in the above paragraph, the vertical emission energy can be estimated as $2.77 \mathrm{eV}$. This value is redshifted by $0.22 \mathrm{eV}$ from the calculated vertical excitation energy $2.99 \mathrm{eV}$ (Table II), and is comparable to the PYP experimental value of 0.26 $\mathrm{eV}$ reported by van der Horst et al. ${ }^{35}$ (vertical excitation 2.78 $\mathrm{eV}$ or $446 \mathrm{~nm}$ versus fluorescence emission $2.52 \mathrm{eV}$ or 492 $\mathrm{nm})$. We note that the redshifting in the gas phase is already $0.21 \mathrm{eV}$, so the dielectric solvation has almost zero effect. Therefore, the redshifting is almost solely determined by vibrational relaxation such as in the gas phase. Why the dielectric (optical at 1.776 and static at 2.0) solvation has almost zero effect on the redshifting? First, the optical dielectricity effects in the vertical excitation and emission processes are almost identical, so they virtually do not contribute to the redshifting. Second, the static dielectric constant used here is 2.0 , which is only slightly larger than 1.776 , and causes a very small amount of molecular polarization energy $(0.027$ $\mathrm{eV}$ as shown above). In short, because the hydrophobic pocket in PYP has a low molecular polarizability (nonpolar), the chromophore will show a redshifting in the fluorescence emission similar to that in the gas phase. This also explains why there is no obvious time-dependent redshifting in the early stage emissions for the chromophore in PYP, as noted by Vengris et al., ${ }^{30}$ because the magnitude of the possible time-dependent redshifting caused by the molecular polarization of the PYP pocket is close to zero. Compared to the PYP pocket, bulk water shows a larger molecular polarization energy (not surprising because water molecules are polar) so the magnitude of the time-dependent redshifting is larger. Of course, the actual pattern of the time-dependent shifting is a separate issue that can hardly be addressed using the current continuum model.

\section{Single bond and double bond twisted structures}

After the initial vibrational relaxation, excited trans-TMpCA ${ }^{-}$may twist $90^{\circ}$ around the $\mathrm{C} 7-\mathrm{C} 8$ single bond and the $\mathrm{C} 8=\mathrm{C} 9$ double bond (Fig. 1). The double bond twisted (DBT) structure may de-excite to the ground state, and form either the trans or cis isomer. The relative energies of the excited state single bond twisted (SBT) and DBT structures are important in characterizing the PES.

The SBT and DBT structures were optimized with the TD-B3LYP method in the gas phase in solutions. In the gas phase, the SBT structure lies $10.25 \mathrm{kcal} / \mathrm{mol}$ below the DBT structure, which lies $22.77 \mathrm{kcal} / \mathrm{mol}$ below the trans (planar) structure. Therefore, upon excitation, the molecule prefers the SBT structure over the DBT and trans structure. As already discussed in Sec. IV C, the trans (planar) structure of the gas phase excited state is actually a saddle point. There is a small barrier (to be discussed more in Sec. IV E) in the 
double bond twisting pathway. These results are similar to those obtained by Groenhof et al., ${ }^{37}$ who found that the single bond twisting barrier (but no barrier in our current work) is lower than the double bond twisting barrier in the gas phase for a QM model that is essentially the trans-TMpCA ${ }^{-}$studied in the current work.

At the gas phase SBT and DBT minima, the excitation energies are 0.48 and $0.39 \mathrm{eV}$, respectively (Table II). Very recently, Boggio-Pasqua et al. optimized the gas phase SBT structure of methyl $p$-coumaric acid $\left(\mathrm{MpCA}^{-}\right.$, differing from $\mathrm{TMpCA}^{-}$by one $\mathrm{S}$ atom) with the $\operatorname{CASSCF}(12,11) / 6-31 \mathrm{G}(\mathrm{d})$ method, and obtained an excitation energy of $1.77 \mathrm{eV}$, which is much higher compared to $0.48 \mathrm{eV}$ in the current study. They refined the excitation energy using single point CASPT2/cc-pVDZ calculations and obtained $1.58 \mathrm{eV}^{42}$ which is still very high. Using their published CASSCF(12,11)/6-31G(d) coordinates of the SBT structure, we performed single point gas phase TD-B3LYP calculations and obtained the following excitation energies: $S_{0} \rightarrow S_{1}$ $\left(\mathrm{HOMO} \rightarrow\right.$ LUMO) $\quad 0.57 \mathrm{eV}$ and $\mathrm{S}_{0} \rightarrow \mathrm{S}_{2} \quad$ (HOMO-1 $\rightarrow$ LUMO) $1.75 \mathrm{eV}$. For the DBT structure, their CASSCF(12,11)/6-31G(d) and CASPT2/cc-pVDZ results are 0.95 and $0.56 \mathrm{eV}$, respectively, as compared to the $0.39 \mathrm{eV}$ in the current work. Using their published CASSCF(12,11)/6$31 \mathrm{G}(\mathrm{d})$ coordinates of the DBT structure, we performed single point TD-B3LYP calculations and obtained the following excitation energies: $\mathrm{S}_{0} \rightarrow \mathrm{S}_{1}(\mathrm{HOMO} \rightarrow \mathrm{LUMO}) 0.34 \mathrm{eV}$ and $\mathrm{S}_{0} \rightarrow \mathrm{S}_{2}$ (HOMO-1 $\rightarrow$ LUMO) $1.89 \mathrm{eV}$. The large differences between the CASSCF and TDDFT results, especially those for the SBT structure, may need further examination.

CPCM solvation significantly changes the absolute and relative energies of the excited state trans, SBT and DBT structures. As the dielectric constant increases from 1 to 2, and then to 78.39 , the absolute energy of the trans structure is lowered by 28.04 and $58.03 \mathrm{kcal} / \mathrm{mol}$; that of the SBT structure is lowered by 16.08 and $31.38 \mathrm{kcal} / \mathrm{mol}$; that of the DBT structure is lowered by 21.28 and $43.42 \mathrm{kcal} / \mathrm{mol}$. The energy lowering in solution is simply the CPCM solvation energy, including a small amount of geometry relaxation energy ongoing from the gas phase to solution. Since the CPCM solvation energy is determined by the charge density of the molecule, the large differences in the CPCM solvation energy between the trans, SBT and DBT structures must be resulted from significant changes in the electron density when the excited molecule changes its geometry. Such charge redistribution in PYP chromophore was noticed and analyzed by Coto et ll. $^{41}$ using the quantum theory of atoms in molecules. Using $\varepsilon=2.0$, the DBT structure lies $5.05 \mathrm{kcal} /$ mol above the SBT structure and $16.01 \mathrm{kcal} / \mathrm{mol}$ below the trans (planar) structure (Table II). Therefore, CPCM solvation can significantly increase the population of the DBT structure, which has roughly $50 \%$ chance to form the ground state cis isomer. The QM/MM and QM simulations of Groenhof et al. ${ }^{37}$ revealed that the initial twisting of the molecule is more likely to be around the single bond in the gas phase, and is more likely to be around the double bond in PYP. In aqueous solution, the DBT structure lies $1.79 \mathrm{kcal} /$ mol below the SBT structure, which lies $8.16 \mathrm{kcal} / \mathrm{mol}$ below the trans (planar) structure (Table II).

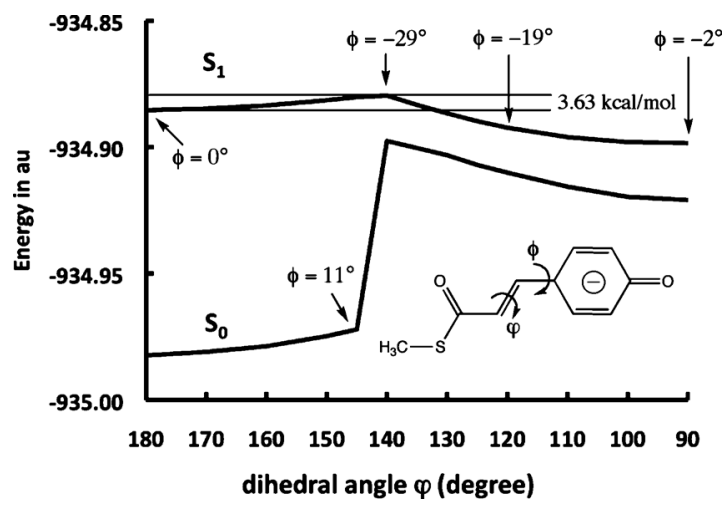

FIG. 2. $\mathrm{S}_{1}$ and $\mathrm{S}_{0}$ potential energy curves in the minimum energy isomerization pathway calculated by fixing the double bond dihedral angle $\varphi$ and optimizing all the other coordinates using CPCM $(\varepsilon=78.39) / T D-B 3 L Y P / a u g-c c-p V D Z$. The peak position of the $S_{1}$ barrier is determined by the interplay between the double bond twisting $(\varphi)$ and the single bond twisting $(\phi)$.

CPCM solvation increases the excitation energies (i.e., ground-excited gaps) of both the SBT and DBT structures, especially that of the SBT structure. As the dielectric constant increases from 1 to 2 and 78.39, the excitation energy of the SBT structure increases sharply from 0.48 to 0.89 and $1.39 \mathrm{eV}$, while that of the DBT structure increases slightly from 0.39 to 0.50 and $0.61 \mathrm{eV}$ (Table II).

\section{E. Double bond isomerization barrier}

The experiment of Vengris et al. suggests that there is a barrier on the excited state PES of trans-TMpCA- in aqueous solution and in PYP (both the wild-type and the Glu46Gln mutant). ${ }^{30}$ The TDDFT calculation of the gas phase excited state potential energy curve along the minimum energy isomerization pathway on the gas phase DFT ground state PES of $\mathrm{TMpCA}^{-}$of Thompson et al. ${ }^{45}$ shows that there is a $5 \mathrm{kcal} / \mathrm{mol}$ barrier. The QM/MM simulation of Groenhof et al. suggests that there is a small barrier in the double bond isomerization pathway on the excited state PES of the anionic form of the chromophore (essentially the trans-TMpCA ${ }^{-}$in the current work) in both the gas phase and PYP. ${ }^{37}$ Coto et al. $^{41}$ determined a minimum energy isomerization pathway for trans- $\mathrm{MpCA}^{-}$in the gas phase using the CASPT2//CASSCF method and found a $3.46 \mathrm{kcal} /$ mol $(0.15 \mathrm{eV})$ barrier. The experiment of Ryan et al. suggests that there is an empirical barrier of $3.4 \mathrm{kcal} / \mathrm{mol}$ in the gas phase isomerization pathway on the $S_{1}$ excited state potential energy surface of trans-p-coumaric acid (trans-pCA), a neutral analog of the PYP chromophore. ${ }^{33}$ Theoretically, Ko et $a{ }^{39}$ and Levine et al. $^{46}$ found a barrier of $2-4 \mathrm{kcal} / \mathrm{mol}$ in the minimum energy isomerization pathway on the gas phase excited state PES of neutral trans-pCA.

We performed a series of constrained geometry optimization for excited trans-TMpCA ${ }^{-}$in aqueous solution using the CPCM/TD-B3LYP method. The C7-C8-C9-C10 dihedral angle $\varphi$ (Figs. 1 and 2) is fixed at $179.9^{\circ}, 170^{\circ}$, $160^{\circ}, 150^{\circ}, 145^{\circ}, 140^{\circ}, 135^{\circ}, 130^{\circ}, 125^{\circ}, 120^{\circ}, 110^{\circ}, 100^{\circ}$, and $91.6^{\circ}$, and all other coordinates are optimized. The $179.9^{\circ}$ and $91.6^{\circ}$ cases correspond to the fully optimized (no symmetry imposed) trans isomer and the DBT structure as 
discussed in previous subsections. Clearly, as shown in Fig. 2 , in aqueous solution, there is a barrier of $3.63 \mathrm{kcal} / \mathrm{mol}$ peaking at $\varphi \sim 140^{\circ}$ (the actual peak might be somewhere between $140^{\circ}$ and $145^{\circ}$ ) on the $S_{1}$ curve. The corresponding $\mathrm{S}_{0}$ ground state energy (at the optimized geometry on $\mathrm{S}_{1}$ ) is also plotted in Fig. 2. It is very interesting that the $S_{0}$ curve increases sharply as the excited state passes the barrier at $\varphi$ $=140^{\circ}$. Inspection of the molecular geometry shows that this is due to a sudden twisting around the $\mathrm{C} 7-\mathrm{C} 8$ single bond: The C6-C7-C8-C9 dihedral angle $\phi$ is $+10.6^{\circ}$ at $\varphi$ $=145^{\circ}$ while it is $-29.1^{\circ}$ at $\varphi=140^{\circ}$. A full examination shows that the optimal values of $\phi$ are $-0.4^{\circ}, 2^{\circ}, 9.7^{\circ}, 9.7^{\circ}$, and $10.6^{\circ}$, respectively, when the $\varphi$ are $179.9^{\circ}, 170^{\circ}, 160^{\circ}$, $150^{\circ}$, and $145^{\circ}$, and are $-29.1^{\circ},-23.5^{\circ},-18.5^{\circ},-12.7^{\circ}$, $-7.3^{\circ}$, and $-2.0^{\circ}$, respectively, when the $\varphi$ are $140^{\circ}, 130^{\circ}$, $120^{\circ}, 110^{\circ}, 100^{\circ}$, and $91.6^{\circ}$. These results indicate that the peak position of the barrier is determined by the interplay of the double bond twisting and the single bond twisting. We also noticed corresponding changes in the position of the hydrogen atom bonded to C8 (Fig. 1), which may indicate the importance of proton tunneling in the isomerization. Passing the barrier, the molecule enters a well on $S_{1}$ with a depth of $11.80 \mathrm{kcal} / \mathrm{mol}(0.51 \mathrm{eV})$.

However, such a minimum energy isomerization pathway cannot be well characterized for the excited trans-TMpCA ${ }^{-}$in the gas phase and a low dielectric solvent ( $\varepsilon=2.0$ ) because the bending of the curve due to the single bond twisting appears at the beginning $\left(\varphi=180^{\circ}\right)$ of the curve in the gas phase and at approximately $\varphi=160^{\circ}$ when $\varepsilon=2.0$. This means that the molecule will optimize to the SBT structure or a similar one while keeping $\varphi$ at $180^{\circ}$ if no or a too weak CPCM solvation is there. Indeed, as already discussed in Sec. IV D, CPCM solvation significantly stabilizes the trans structure over the SBT and DBT ones, making the trans structure a minimum point on the excited state PES. The SBT structure is very likely the global minimum on the PESs in the gas phase and a low dielectric solvent $(\varepsilon=2.0)$ although we could not do an exhaustive global search. It is emphasized here that although such a minimum energy pathway cannot be characterized using TD-B3LYP geometry optimization, it does not necessarily mean that there is no such a pathway (though not a minimum energy one) in the gas phase or a low dielectric solvent. Actually we believe that the isomerization pathways in various environments should be similar to each other, and the one characterized using the CPCM $(\varepsilon=78.39) / T D-B 3 L Y P$ method can be used as a representative. To at least obtain some estimates for the cases in the gas phase and protein, single point TD-B3LYP and CPCM $(\varepsilon=2.0) /$ TD-B3LYP calculations were performed for four points on the minimum energy curve obtained with CPCM $(\varepsilon=78.39) / T D-B 3 L Y P$ geometry optimization, i.e., the ones with $\varphi=179.9^{\circ}, 145^{\circ}, 140^{\circ}, 91.6^{\circ}$. These calculations show that when $\varepsilon=1$ and 2 , the peak of the barrier tends to be at $\varphi \sim 145^{\circ}$ (we did not perform a finer search). As the dielectric constant increases from 1 to 2.0, and then to 78.39, the excited state barrier changes from 5.16 to 3.29, and then to $3.63 \mathrm{kcal} / \mathrm{mol}$, the excited state well depth decreases from 20.20 to 16.06 , and then to $11.80 \mathrm{kcal} / \mathrm{mol}$. Clearly, CPCM solvation tends to decrease the barrier and well depth. The $5.16 \mathrm{kcal} / \mathrm{mol}(0.22 \mathrm{eV})$ barrier obtained in the gas phase calculation compares well to the $\sim 5 \mathrm{kcal} / \mathrm{mol}$ value obtained by Thompson et $a l^{45}$ for the gas phase $\mathrm{TMpCA}^{-}$using a TDDFT method, and can be compared to the $3.46 \mathrm{kcal} / \mathrm{mol}(0.15 \mathrm{eV})$ value obtained by Coto et al. ${ }^{41}$ for the gas phase $\mathrm{MpCA}^{-}$using CASPT2//CASSCF method. The $20.20 \mathrm{kcal} / \mathrm{mol}(0.88 \mathrm{eV})$ deep well obtained in the gas phase calculation compares very well with the $\sim 1 \mathrm{eV}$ value estimated by Lee et $a l .{ }^{36}$ from real-time observation of the gas phase dynamics of $\mathrm{MpCA}^{-}$, for which Coto et al. ${ }^{41}$ obtained $0.17 \mathrm{eV}$ using CASPT2//CASSCF.

\section{CONCLUSION}

To conclude, we highlight the following results and findings.

(1) The CPCM/TDDFT analytic gradient is derived and implemented in GAMESS. Using the FIXPVA tessellation scheme, rigorously continuous and smooth potential energy surfaces, as well as exact analytic gradient, are obtained.

(2) The gas phase vertical excitation energy predicted by TD-B3LYP for trans-TMpCA ${ }^{-}$is $0.5 \mathrm{eV}$ too high as compared to experiment. The aqueous vertical excitation energy predicted by CPCM/TD-B3LYP is shifted by $-0.1 \mathrm{eV}$, while the experimentally determined shift is approximately $0.44 \mathrm{eV}$.

(3) $\mathrm{CPCM} / \mathrm{TD}-\mathrm{B} 3 \mathrm{LYP}$ predicts that the vertical emission maximum of trans-TMpCA ${ }^{-}$at $\sim 2$ ps is likely to be redshifted by $0.67 \mathrm{eV}$ as compared to the vertical absorption maximum, in good agreement with the experimental value of $0.74 \mathrm{eV}$.

(4) $\mathrm{CPCM} / \mathrm{TD}-\mathrm{B} 3 \mathrm{LYP}$ predicts that the early stage fluorescence emission maximum of the PYP chromophore is likely to be redshifted by $0.22 \mathrm{eV}$ as compared to the vertical absorption maximum, in good agreement with the experimental value of $0.26 \mathrm{eV}$. In addition, the calculations also indicate that this redshifting shows a negligible time-dependent change.

(5) In the gas phase, excited trans-TMpCA- tends to form (with no barrier) the single bond twisted structure. CPCM solvation preferentially stabilizes the double bond twisted structure, and should significantly increase the yield of the cis isomer.

(6) The minimum energy pathway of the double bond isomerization was obtained using CPCM $(\varepsilon=78.39) / T D-B 3 L Y P$ geometry optimization. In aqueous solution there is a barrier of $3.63 \mathrm{kcal} / \mathrm{mol}(0.16$ $\mathrm{eV})$ and a well with a depth of $11.80 \mathrm{kcal} / \mathrm{mol}(0.51$ $\mathrm{eV}$ ). Since the gas phase minimum energy pathway cannot be well characterized, single point energies were obtained on the aqueous pathway. In the gas phase, the barrier height is $\sim 5.2 \mathrm{kcal} / \mathrm{mol}(0.23 \mathrm{eV})$. The well depth is $\sim 20 \mathrm{kcal} / \mathrm{mol}(0.88 \mathrm{eV})$, in good agreement with an experimental value of $\sim 1 \mathrm{eV}$. 


\section{ACKNOWLEDGMENTS}

This work was supported by start-up funds from the University of Nebraska-Lincoln.

${ }^{1}$ M. E. Casida, in Recent Advances in Density Functional Methods, edited by D. P. Chong (World Scientific, Singapore, 1995), pp. 155; M. E. Casida, C. Jamorski, K. C. Casida, and D. R. Salahub, J. Chem. Phys. 108, 4439 (1998)

${ }^{2}$ T. Ziegler, M. Seth, M. Krykunov, J. Autschbach, and F. Wang, J. Chem. Phys. 130, 154102 (2009).

${ }^{3}$ C. Van Caillie and R. D. Amos, Chem. Phys. Lett. 308, 249 (1999); 317, 159 (2000).

${ }^{4}$ F. Furche and R. Ahlrichs, J. Chem. Phys. 117, 7433 (2002); 121, 12772 (2004).

${ }^{5}$ N. C. Handy and H. F. Schaefer III, J. Chem. Phys. 81, 5031 (1984).

${ }^{6}$ A. Öhrn and G. Karlstrom, Mol. Phys. 104, 3087 (2006).

${ }^{7}$ Y. Lin and J. Gao, J. Chem. Theory Comput. 3, 1484 (2007).

${ }^{8}$ J. Kongsted, A. Osted, K. V. Mikkelsen, and O. Christiansen, J. Chem. Phys. 118, 1620 (2003); J. Kongsted, A. Osted, K. V. Mikkelsen, P.-O. Astrand, and O. Christiansen, ibid. 121, 8435 (2004).

${ }^{9}$ L. Jensen, P. T. van Duijnen, and J. G. Snijders, J. Chem. Phys. 119, 3800 (2003); P. T. Van Duijnen and T. L. Netzel, J. Phys. Chem. A 110, 2204 (2006); C. R. Jacob, J. Neugebauer, L. Jensen, and L. Visscher, Phys. Chem. Chem. Phys. 8, 2349 (2006).

${ }^{10}$ S. Yoo, F. Zahariev, S. Sok, and M. S. Gordon, J. Chem. Phys. 129, 144112 (2008)

${ }^{11}$ M. Cossi and V. Barone, J. Chem. Phys. 115, 4708 (2001).

${ }^{12}$ G. Scalmani, M. J. Frisch, B. Mennucci, J. Tomasi, R. Cammi, and V. Barone, J. Chem. Phys. 124, 094107 (2006).

${ }^{13}$ S. Miertuš, E. Scrocco, and J. Tomasi, Chem. Phys. 55, 117 (1981).

${ }^{14}$ E. Cancès, B. Mennucci, and J. Tomasi, J. Chem. Phys. 107, 3032 (1997).

${ }^{15}$ B. Mennucci, E. Cances, and J. Tomasi, J. Phys. Chem. B 101, 10506 (1997).

${ }^{16}$ V. Barone and M. Cossi, J. Phys. Chem. A 102, 1995 (1998).

${ }^{17} \mathrm{H}$. Li and J. H. Jensen, J. Comput. Chem. 25, 1449 (2004).

${ }^{18}$ A. Klamt and G. Schuurmann, J. Chem. Soc., Perkin Trans. 2 2, 799 (1993)

${ }^{19}$ T. N. Truong and E. V. Stefanovich, Chem. Phys. Lett. 240, 253 (1995).

${ }^{20}$ D. M. Chipman, Theor. Chem. Acc. 107, 80 (2002); D. M. Chipman and M. Dupuis, ibid. 107, 90 (2002).

${ }^{21}$ A. V. Marenich, R. M. Olson, C. P. Kelly, C. J. Cramer, and D. G. Truhlar, J. Chem. Theory Comput. 3, 2011 (2007).

${ }^{22}$ P. Su and H. Li, J. Chem. Phys. 130, 074109 (2009).

${ }^{23}$ A. W. Lange and J. M. Herbert, The Journal of Physical Chemistry Letters $\mathbf{1}, 556(2010)$

${ }^{24}$ G. Scalmani and M. J. Frisch, J. Chem. Phys. 132, 114110 (2010).

${ }^{25}$ T. E. Meyer, Biochim. Biophys. Acta 806, 175 (1985).

${ }^{26}$ J. J. Van Beeumen, B. V. Devreese, S. M. Van Bun, W. D. Hoff, K. J. Hellingwerf, T. E. Meyer, D. E. McRee, and M. A. Cusanovich, Protein Sci. 2, 1114 (1993); M. Baca, G. E. O. Borgstahl, M. Boissinot, P. M. Burke, D. R. Williams, K. A. Slater, and E. D. Getzoff, Biochemistry 33, 14369 (1994).

${ }^{27}$ U. K. Genick, S. M. Soltis, P. Kuhn, I. L. Canestrelli, and E. D. Getzoff, Nature (London) 392, 206 (1998).

${ }^{28}$ D. S. Larsen, M. Vengris, I. H. M. van Stokkum, M. A. van der Horst, R. A. Cordfunke, K. J. Hellingwerf, and R. van Grondelle, Chem. Phys. Lett. 369, 563 (2003).

${ }^{29}$ D. S. Larsen, M. Vengris, I. H. M. van Stokkum, M. A. van der Horst, F. L. de Weerd, K. J. Hellingwerf, and R. van Grondelle, Biophys. J. 86, 2538 (2004)

${ }^{30}$ M. Vengris, M. A. van der Horst, G. Zgrabli, I. H. M. van Stokkum, S.
Haacke, M. Chergui, K. J. Hellingwerf, R. van Grondelle, and D. S. Larsen, Biophys. J. 87, 1848 (2004).

${ }^{31}$ M. Vengris, D. S. Larsen, M. A. van der Horst, O. F. A. Larsen, K. J. Hellingwerf, and R. van Grondelle, J. Phys. Chem. B 109, 4197 (2005); L. L. Premvardhan, F. Buda, M. A. van der Horst, D. C. Luhrs, K. J. Hellingwerf, and R. van Grondelle, ibid. 108, 5138 (2004).

${ }^{32}$ I. B. Nielsen, S. Boye-Peronne, M. O. A. El Ghazaly, M. B. Kristensen, S. Brondsted Nielsen, and L. H. Andersen, Biophys. J. 89, 2597 (2005).

${ }^{33}$ W. L. Ryan, D. J. Gordon, and D. H. Levy, J. Am. Chem. Soc. 124, 6194 (2002).

${ }^{34}$ P. Changenet-Barret, P. Plaza, and M. M. Martin, Chem. Phys. Lett. 336, 439 (2001); A. Espagne, P. Changenet-Barret, P. Plaza, and M. M. Martin, J. Phys. Chem. A 110, 3393 (2006).

${ }^{35}$ M. A. van der Horst, J. C. Arents, R. Kort, and K. J. Hellingwerf, Photochem. Photobiol. Sci. 6, 571 (2007).

${ }^{36}$ I. R. Lee, W. Lee, and A. H. Zewail, Proc. Natl. Acad. Sci. U.S.A. 103, 258 (2006).

${ }^{37}$ G. Groenhof, M. Bouxin-Cademartory, B. Hess, S. P. de Visser, H. J. C. Berendsen, M. Olivucci, A. E. Mark, and M. A. Robb, J. Am. Chem. Soc. 126, 4228 (2004)

${ }^{38}$ V. Molina and M. Merchan, Proc. Natl. Acad. Sci. U.S.A. 98, 4299 (2001).

${ }^{39}$ C. Ko, B. Levine, A. Toniolo, L. Manohar, S. Olsen, H.-J. Werner, and T. J. Martinez, J. Am. Chem. Soc. 125, 12710 (2003).

${ }^{40}$ E. V. Gromov, I. Burghardt, H. Koppel, and L. S. Cederbaum, J. Am. Chem. Soc. 129, 6798 (2007).

${ }^{41}$ P. B. Coto, D. Roca-Sanjuan, L. Serrano-Andres, A. Martin-Pendas, S. Marti, and J. Andres, J. Chem. Theory Comput. 5, 3032 (2009).

${ }^{42}$ M. Boggio-Pasqua, M. A. Robb, and G. Groenhof, J. Am. Chem. Soc. 131, 13580 (2009)

${ }^{43}$ A. Sergi, M. Gruning, M. Ferrario, and F. Buda, J. Phys. Chem. B 105, 4386 (2001)

${ }^{44}$ G. Groenhof, M. F. Lensink, H. J. C. Berendsen, J. G. Snijders, and A. E. Mark, Proteins 48, 202 (2002).

${ }^{45}$ M. J. Thompson, D. Bashford, L. Noodleman, and E. D. Getzoff, J. Am. Chem. Soc. 125, 8186 (2003).

${ }^{46}$ B. G. Levine, C. Ko, J. Quenneville, and T. J. Martinez, Mol. Phys. 104, 1039 (2006)

${ }^{47}$ M. Chiba, D. G. Fedorov, and K. Kitaura, J. Comput. Chem. 29, 2667 (2008).

${ }^{48}$ B. Mennucci, R. Cammi, and J. Tomasi, J. Chem. Phys. 109, 2798 (1998); M. Cossi and V. Barone, ibid. 112, 2427 (2000).

${ }^{49}$ M. Cossi, N. Rega, G. Scalmani, and V. Barone, J. Comput. Chem. 24, 669 (2003).

${ }^{50}$ M. W. Schmidt, K. K. Baldridge, J. A. Boatz, S. T. Elbert, M. S. Gordon, J. H. Jensen, S. Koseki, N. Matsunaga, K. A. Nguyen, S. J. Su, T. L. Windus, M. Dupuis, and J. A. Montgomery, J. Comput. Chem. 14, 1347 (1993); M. S. Gordon and M. W. Schmidt, in Theory and Applications of Computational Chemistry, edited by C. E. Dykstra, G. Frenking, K. S. Kim, and G. E. Scuseria (Elsevier, New York, 2005).

${ }^{51}$ M. Chiba, T. Tsuneda, and K. Hirao, J. Chem. Phys. 124, 144106 (2006); Chem. Phys. Lett. 420, 391 (2006).

${ }^{52}$ J. Tomasi, B. Mennucci, and E. Cances, J. Mol. Struct.: THEOCHEM 464, 211 (1999).

${ }^{53}$ H. Li, C. S. Pomelli, and J. H. Jensen, Theor. Chem. Acc. 109, 71 (2003).

${ }^{54}$ R. H. Hertwig and W. Koch, Chem. Phys. Lett. 268, 345 (1997).

${ }^{55}$ T. H. Dunning, J. Chem. Phys. 90, 1007 (1989).

${ }^{56}$ C. S. Pomelli, J. Tomasi, and V. Barone, Theor. Chem. Acc. 105, 446 (2001).

${ }^{57}$ E. M. González, L. Guidoni, and C. Molteni, Phys. Chem. Chem. Phys. 11, 4556 (2009).

${ }^{58}$ Y. Ma, M. Rohlfing, and C. Molteni, J. Chem. Theory Comput. 6, 257 (2010).

${ }^{59}$ M. Chiba, D. G. Fedorov, and K. Kitaura, Chem. Phys. Lett. 444, 346 (2007). 